Chez l'homme, les anomalies du génome mitochondrial donnent le plus souvent naissance à un syndrome myopathique, avec souvent atteinte des muscles oculaires. Dans le pancréas, c'est plutôt la fonction externe qui est altérée sous forme du syndrome de Pearson ( $m / s n^{\circ} 7$, vol. 5, p. 459 et 472). L'équipe de D.C. Wallace (Atlanta, GE, USA), habituée des "premières " en pathologie des mitochondries, a étudié [1] une famille dont neuf membres suivis sur trois générations ont présenté un diabète et/ou une surdité. La grand-mère a eu des enfants atteints, de deux pères différents; sa fille a transmis la maladie à la petite-fille ; son fils, atteint lui aussi, n'a pas été transmetteur. Aucune anomalie clinique musculaire ni aucun ptosis n'ont été notés dans la famille. L'analyse biochimique des mitochondries isolées à partir du muscle montra une baisse considérable de tous les complexes de phosphorylation oxydative. La découverte la plus importante fut celle de l' "hétéroplasmie " de l'ADN mitochondrial (mt), témoignant de l'existence de deux populations différentes de mitochondries : $31 \%$ de l'ADNmt étaient constitués d'ADN normal, alors que $69 \%$ portaient une délétion considérable, amputant l'ADN de plus de $10 \mathrm{~kb}$ (entre les nucléotides 4398 - à l'intérieur de l'ARNt Gln - et 14822 - dans le gène du cytochrome b). La région de la boucle de développement était conservée ainsi que l'origine de réplication du brin lourd ; mais, ce qui est exceptionnel dans les délétions de l'ARNmt, l'origine OL du brin léger était incluse dans la délétion. Chez six membres de la famille en lignée maternelle, la même délétion, dans des proportions semblables, fut retrouvée à partir des leucocytes et des plaquettes, mais non chez un descendant en lignée paternelle. Plusieurs points distinguent cette observation de toutes celles qui étaient connues antérieurement.

- Sur le plan clinique, diabète et surdité restent isolés, et ne sont pas accompagnés des anomalies classiques musculaires, cliniques ou anatomiques, malgré l'effondrement des phosphorylations oxydatives mesurées in vitro.

- La délétion de $10,5 \mathrm{~kb}$ a été trouvée, identique de taille et de limites ainsi qu'en proportions semblables, chez tous les membres de la famille en lignée maternelle. Cela constraste avec l'apparition sporadique habituelle ou, dans les formes familiales, les variations des délétions d'un sujet à un autre [2]. Lorsque la délétion est la même chez plusieurs membres d'une même famille, c'est la proportion qui varie : chez les apparentés sains, on ne peut la mettre en évidence que par amplification de l'ADN [3]. Il en va de même des formes à hérédité autosomique, dont la transmission est nucléaire ( $m / s n^{\circ} 2$, vol. 7, p. 172), qui sont porteuses de délétions multiples [4].

- Enfin, alors que toutes les délétions connues respectaient les deux origines de réplication des brins, l'origine $\mathrm{OL}$ est ici perdue. Cela pose problème, car si l'on se réfère aux travaux biochimiques, on admet que OL est essentiel à la réplication de l'ADNmt chez les mammifères [5]. On est tenté de rattacher à cette perte de OL les particularités de cette maladie ; en particulier le maintien des ADNmt délétés dans le muscle à un niveau qui reste compatible avec un fonctionnement normal de ce tissu, et qui ne se modifie pas d'une génération à la suivante.

\title{
口曰 BRÈVES
}

La lésion moléculaire de la myopathie du chien. On connaît depuis quelques années une dystrophie musculaire apparue dans une souche de labrador (golden retriever). C'est le meilleur modèle animal de la myopathie de Duchenne, par son aspect clinique et sa gravité, qui contraste avec la bénignité du modèle de la souris $m d x$. Comme dans les maladies de l'homme et de la souris, la protéine dystrophine est absente. Une équipe américaine (11 auteurs) vient d'en élucider la lésion moléculaire. La mutation d'une seule base, au site de consensus 3' de l'intron 6 provoque l'élimination de l'exon 7, et, par suite d'un décalage de phase, l'apparition d'un codon de terminaison dans l'exon 8. La protéine est donc tronquée et est absente du muscle. Cette anomalie semble être le premier exemple d'absence de dystrophine due à une mutation ponctuelle dans un site d'épissage, en dehors des cas de délétion.

[1. Sharp JH, et al. Genomics 1992 ; 13 : 115-21.]

प Mutation non-sens du gène de la glucokinase dans une forme de diabète non-insulinodépendant. Nous avons rapporté dans un flash publié en mars ( $m / s n^{\circ} 3$, vol. $\left.8, p .297\right)$ que l'équipe du CEPH (centre d'étude du polymorphisme humain) avait identifié le gène de glucokinase comme étant très probablement impliqué dans un grand nombre d'observations de diabètes non-insulinodépendants du sujet jeune (MODY, maturity onset diabeles of the youzg). Ces données ont depuis été confirmées par une équipe de chercheurs anglais et américains [1]. Nos prédictions qu'une confirmation n'allait pas tarder à arriver sous la formc: de la détection de mutations de ce gène n'a pas mis longtemps avant de se vérifier. Dans le numéro du 23 avril 1992 de Nature, la même équipe du CEPH, dirigée par P.H. Frogel et D. Cohen, en collaboration avec une équipe américaine de Chicago (IL, USA), vient d'observer, dans une famille, la présence d'une mutation non-sens changeant l'acide glutamique 279 en un codon-stop [2]. Il est sûr que $\mathrm{N}$. Vionnet $e t$ al., les auteurs de cet article, ont également entrepris la caractérisation des anomalies moléculaires dans d'autres familles atteintes de ce type de diabète. Leurs homogénéité ou hétérogénéité donnera des indications sur l'origine génétique de cette maladie, événements récurrents aléatoires ou effet fondateur.

[1. Hattersley AT, et al. Lancet 1992 ; 339 : 1307-10.]

[2. Vionnet N, et al. Nature 1992 ; 356 : 721-2.] 